\section{Polyomaviruses KI and WU in Immuno- compromised Patients with Respiratory Disease}

\author{
Thomas Mourez, Anne Bergeron, Patricia Ribaud, \\ Catherine Scieux, Régis Peffault de Latour, \\ Abdellatif Tazi, Gérard Socié, François Simon, \\ and Jérôme LeGoff
}

Polyomaviruses KI (KIPYV) and WU (WUPyV) were recently identified, mainly in respiratory specimens from children. Among 200 patients with respiratory disorders admitted to Saint Louis Hospital, Paris, France, KIPyV was detected in $8 \%$ and WUPyV in 1\%. KIPyV was significantly more frequent among human stem cell transplant patients (17.8\% vs. $5.1 \% ; p=0.01)$.

$\mathrm{R}$ ecently, 2 new, distinct polyomaviruses (PyVs), KI (KIPyV) and WU (WUPyV), were identified in respiratory specimens, mainly from children $<5$ years of age with respiratory tract infections. The first retrospective studies of respiratory specimens in Sweden and Australia showed a KIPyV prevalence of $1 \%$ and $2.5 \%$, respectively $(1,2)$. Studies conducted in Australia and the United States showed a WUPyV prevalence in respiratory specimens of $3 \%$ and $0.7 \%$, respectively (3). Further studies conducted in Canada and South Korea have shown similar frequencies $(4,5)$. In this study, we examined the prevalence of KIPyV and WUPyV in immunocompromised patients with suspected respiratory tract infections.

\section{The Study}

From January through June 2007, 265 respiratory samples were received in the laboratory of Saint Louis Hospital, Paris: 154 nasal aspirates (NA) and 111 bronchoalveolar lavage (BAL) specimens collected from 200 patients with suspected upper or lower respiratory tract infections. This hospital specializes in the management of immunocompromised patients. Respiratory samples were collected for the diagnosis of acute respiratory illness; $89 \%$ of samples were from immunocompromised patients. Their median age was 46 years (range 3.6-85.3 years). Given the observational nature of the study, French law did not require ethical approval or informed consent.

Author affiliation: Hôpital Saint Louis, Paris, France

DOI: 10.3201/1501.080758
The specimens were routinely tested for influenza A and $\mathrm{B}$ viruses, respiratory syncytial virus, and parainfluenza viruses 1, 2, and 3 by immunofluorescence assay (Imagen; DakoCytomation, Trappes, France). Specimens positive for KIPyV or WUPyV were tested for adenoviruses; human bocavirus; human rhinoviruses; human metapneumovirus; human coronaviruses OC43, 229E, NL63, HKU1; and human PyVs BK and JC by using PCR methods (6-11). Total nucleic acid was extracted from $200 \mu \mathrm{L}$ of NA, BAL, or stool specimens by using the EasyMag System (bioMérieux, Marcy l'Etoile, France). KIPyV was detected with an in-house real-time PCR assay targeting the VP1 gene. The primers and hydrolysis probe were designed by using Primer Express 3.0 software (Applied Biosystems, Foster City, CA, USA). The final reaction volume was $25 \mu \mathrm{L}$ and contained $12.5 \mathrm{pmol}$ of SLKI-VP1s $\left(5^{\prime}-\right.$ GGAAATACAGCTGCTCAGGAT-3') and SLKI-VP1as (5'-CTTTGATACTTGAACCGCTTTCCTT-3'), 6.25 pmol of corresponding probe SLKI-VP1PR (5'-6FAM-C GTGACCCCACCCCTCATTACTGGTC-TAMRA-3'), $12.5 \mu \mathrm{L}$ of TaqMan Universal Master Mix (Applied Biosystems), and $5 \mu \mathrm{L}$ of DNA extract. The reaction was run on a 7500 Real-Time PCR System (Applied Biosystems). The specificity of positive specimens was confirmed by using PCR and nested PCR with primers POLVP1-39F/POLVP1363R and POLVP1-118F/POLVP1-324R, as described (1). The PCR products were then sequenced and compared with the previously described sequences from Sweden and Australia (GenBank accession nos. EF127906, EF127907, EF127908, EF520287, EF520288, and EF520289). WUPyV was detected by PCR as described (3). PCR products with the expected molecular weights were sequenced by using primers AG0044 and AG0045 and compared to published sequences (GenBank accession nos. EF444550, EF444551, EF444552, EF444553, and EF444554) (3).

KIPyV was detected in $17(6.5 \%)$ of the 265 respiratory samples and in $16(8.0 \%)$ of the 200 patients. All cases were confirmed by a nested PCR targeting another region of the VP1 gene. Twelve of the 17 PCR products were successfully sequenced, and all shared $100 \%$ homology with published sequences. WUPyV was detected in only 2 patients (1.0\%). Genome sequencing showed $98 \%$ homology with reported WUPyV sequences.

Six KIPyV-positive patients (37.5\%) had co-infections with other respiratory viruses, and 2 of them (12.5\%) had a pulmonary bacterial infection (online Appendix Table, available from www.cdc.gov/EID/content/15/1/107-appT. $\mathrm{htm})$. One WUPyV-infected patient who exhibited acute respiratory failure had concomitant pneumonia caused by Pseudomonas aeruginosa infection. None of the 15 patients who were positive for KIPyV or WUPyV and tested for fungi had respiratory or blood samples positive for Aspergillus spp. 
The clinical characteristics of the patients with KIPyV or WUPyV infection and their general outcome until December 2007 are given in the online Appendix Table. All but 3 had well-identified systemic immunosuppression. However, all had severe coexisting conditions. All KIPyV- and WUPyVpositive patients had acute respiratory disorders.

Eight KIPyV-positive patients had received allogeneic stem cell transplants; 5 of them had exhibited profound neutropenia in the 2 weeks before the respiratory sample was found to be positive. The detection of KIPyV was significantly more frequent among hematopoietic stem cell transplant (HSCT) recipients than among other patients $(17.8 \%$ [8/45] vs. $5.1 \%$ [8/155], $\mathrm{p}=0.01)$ (Table). Lung or sinus imaging was assessed by computed tomography scan for 12 KIPyV-positive patients. Lung parenchyma abnormalities were noted in 9 patients, and sinusitis was diagnosed for 2 patients.

Taking into consideration both the frequency of digestive symptoms in our patients and the former published detection of KIPyV in a stool sample, we looked for KIPyV infection in the available stool samples while the respiratory samples were being assessed for KIPyV (1). Strikingly, 3 of 4 samples tested were positive for KIPyV (with cycle threshold values of 35.0, 38.3, and 40.3). All were collected from HSCT recipients. In addition, 1 HSCT patient who experienced diarrhea demonstrated persistent excretion of KIPyV in 9 consecutive stool specimens collected between June and the end of November 2007 (data not shown).

\section{Conclusions}

This study shows the prevalence of KIPyV and WUPyV among immunocompromised patients with respiratory disorders. Previously, these 2 viruses had been observed mainly in young children (1-3). Of the few adult patients with KIPyV or WUPyV infection mentioned in these studies, most were immunocompromised $(3,12)$.

Considering the seemingly higher prevalence of KIP$\mathrm{yV}$ in our population (8\%), immunocompromised patients may be more susceptible to this $\mathrm{PyV}$, as they are to $\mathrm{JC}$ and BK viruses (13-15). Results from previous reports suggest a similar frequency of both KIPyV and WUPyV infections being found in respiratory specimens, ranging from $1 \%$ to $3 \%$. In contrast, in our series, we found a likely difference between the prevalence of KIPyV (8\%) and WUPyV (1\%), which suggests that the replication or reactivation of the 2 viruses in the respiratory tract may differ between immunocompromised and immunocompetent patients. However, this difference requires further investigation, in particular, by using similar real-time PCR assays. Notably, a significantly higher prevalence of KIPyV infection was found among HSCT patients, which suggests that a profound $\mathrm{T}$-cell deficiency may be a factor in facilitating KIPyV replication.
Table. Frequency of $\mathrm{KI}$ polyomavirus detection in respiratory samples*

\begin{tabular}{|c|c|c|c|}
\hline \multirow[b]{2}{*}{ Sample source } & \multicolumn{2}{|c|}{$\begin{array}{l}\text { KIV detection in respiratory } \\
\text { samples, no. (\%) }\end{array}$} & \multirow[b]{2}{*}{ Total } \\
\hline & Positive & Negative & \\
\hline HSCT patients & $8(17.8) \dagger$ & 37 (82.2) & 45 \\
\hline Other patients & $8(5.2)$ & 147 (94.8) & 155 \\
\hline Total & 16 & 184 & 200 \\
\hline
\end{tabular}

As reported in other populations, our patients who yielded positive specimens for KIPyV or WUPyV had conditions ranging from a common cold to acute respiratory distress that required invasive ventilation. Respiratory coinfections, observed in other studies, had likely accounted for at least some clinical features. In the 7 of our patients in whom KIPyV was the sole pathogen detected in the respiratory tract, despite comprehensive screening for viruses, bacteria, parasites, and fungi, clinical and radiographic patterns were varied. Some of the patients had only upper respiratory tract infections, notably sinusitis, whereas others had lung parenchyma abnormalities as defined by computed tomographic scan imaging. However, due to the retrospective nature of the study, and therefore the lack of a control group of immunocompromised patients without respiratory symptoms, the association of KIPyV infection with the occurrence of respiratory disease cannot be stated definitively.

In conclusion, the seemingly higher frequency of KIP$\mathrm{yV}$ shedding in immunocompromised patients (as observed with other PyVs) and the detection of KIPyV as a single pathogen in respiratory disease (e.g., as cytomegalovirus recurrence can lead to pneumonia in immunocompromised patients) together support a reactivation hypothesis. Nevertheless, a reinfection hypothesis cannot be excluded due to immunocompromised patients' increased risk of acquiring viral infection from exogenous sources

Controlled prospective studies of KIPyV shedding before and during immunosuppression will help determine the pathogenic role of this virus. The clinical implication of $\mathrm{KIPyV}$ detection in stools and the mechanisms underlying the concomitant presence in gastrointestinal and respiratory tracts also deserve further analysis.

\section{Acknowledgments}

We thank F. Freymuth, P. Lebon, and A. Vabret for advice and technical assistance and for providing positive virus controls.

Dr Mourez is a virologist in the Laboratory of Microbiology, Saint Louis University Hospital, Paris. His research interests include the development of tests for the diagnosis of emerging respiratory viruses and the study of the circulation and molecular analysis of human respiratory viruses in pediatric and immunocompromised patients. 


\section{References}

1. Allander T, Andreasson K, Gupta S, Bjerkner A, Bogdanovic G, Persson MA, et al. Identification of a third human polyomavirus. J Virol. 2007;81:4130-6. DOI: 10.1128/JVI.00028-07

2. Bialasiewicz S, Whiley DM, Lambert SB, Wang D, Nissen MD, Sloots TP. A newly reported human polyomavirus, KI virus, is present in the respiratory tract of Australian children. J Clin Virol. 2007;40:15-8. DOI: 10.1016/j.jcv.2007.07.001

3. Gaynor AM, Nissen MD, Whiley DM, Mackay IM, Lambert SB, Wu $\mathrm{G}$, et al. Identification of a novel polyomavirus from patients with acute respiratory tract infections. PLoS Pathog. 2007;3:e64. DOI: 10.1371/journal.ppat.0030064

4. Abed Y, Wang D, Boivin G. WU polyomavirus in children, Canada. Emerg Infect Dis. 2007;13:1939-41.

5. Han TH, Chung JY, Koo JW, Kim SW, Hwang ES. WU polyomavirus in children with acute lower respiratory tract infections, South Korea. Emerg Infect Dis. 2007;13:1766-8.

6. Robin M, Marque-Juillet S, Scieux C, Peffault de Latour R, Ferry C, Rocha $\mathrm{V}$, et al. Disseminated adenovirus infections after allogeneic hematopoietic stem cell transplantation: incidence, risk factors and outcome. Haematologica. 2007;92:1254-7. DOI: 10.3324/haematol. 11279

7. Savolainen C, Mulders MN, Hovi T. Phylogenetic analysis of rhinovirus isolates collected during successive epidemic seasons. Virus Res. 2002;85:41-6. DOI: 10.1016/S0168-1702(02)00016-3

8. Allander T, Tammi MT, Eriksson M, Bjerkner A, Tiveljung-Lindell A, Andersson B. Cloning of a human parvovirus by molecular screening of respiratory tract samples. Proc Natl Acad Sci U S A. 2005;102:12891-6. DOI: 10.1073/pnas.0504666102

9. Bellau-Pujol S, Vabret A, Legrand L, Dina J, Gouarin S, PetitjeanLecherbonnier J, et al. Development of three multiplex RT-PCR assays for the detection of 12 respiratory RNA viruses. J Virol Methods. 2005;126:53-63. DOI: 10.1016/j.jviromet.2005.01.020
10. Vabret A, Dina J, Gouarin S, Petitjean J, Corbet S, Freymuth F. Detection of the new human coronavirus HKU1: a report of 6 cases. Clin Infect Dis. 2006;42:634-9. DOI: 10.1086/500136

11. Herman J, Van Ranst M, Snoeck R, Beuselinck K, Lerut E, Van Damme-Lombaerts R. Polyomavirus infection in pediatric renal transplant recipients: evaluation using a quantitative real-time PCR technique. Pediatr Transplant. 2004;8:485-92. DOI: 10.1111/j.13993046.2004.00211.x

12. Norja P, Ubillos I, Templeton K, Simmonds P. No evidence for an association between infections with WU and KI polyomaviruses and respiratory disease. J Clin Virol. 2007;40:307-11. Epub 2007 Nov 7. DOI: $10.1016 /$ j.jcv.2007.09.008

13. Shapiro S, Robin M, Esperou H, Devergie A, Rocha V, Garnier F, et al. Polyomavirus nephropathy in the native kidneys of an unrelated cord blood transplant recipient followed by a disseminated polyomavirus infection. Transplantation. 2006;82:292-3. DOI: 10.1097/01. tp.0000226172.68372.f9

14. Hirsch HH. BK virus: opportunity makes a pathogen. Clin Infect Dis. 2005;41:354-60. DOI: 10.1086/431488

15. Drachenberg CB, Hirsch HH, Papadimitriou JC, Gosert R, Wali RK, Munivenkatappa R, et al. Polyomavirus BK versus JC replication and nephropathy in renal transplant recipients: a prospective evaluation. Transplantation. 2007;84:323-30. DOI: 10.1097/01. tp.0000269706.59977.a5

Address for correspondence: Jérôme LeGoff, Laboratoire de Microbiologie, Hôpital Saint-Louis 1, Ave Claude Vellefaux 75010 Paris, France; email: jerome.le-goff@sls.aphp.fr

All material published in Emerging Infectious Diseases is in the public domain and may be used and reprinted without special permission; proper citation, however, is required.

\section{EMERGING

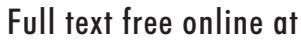 www.cdc.gov/eid

The print journal is available at no charge to public health professionals

$\square$ YES, I would like to receive Emerging Infectious Diseases.

Please print your name and business address in the box and return by fax to 404-639-1954 or mail to

EID Editor

CDC/NCID/MS D61

1600 Clifton Road, NE

Atlanta, GA 30333

Moving? Please give us your new address (in the box) and print the number of your old mailing label here

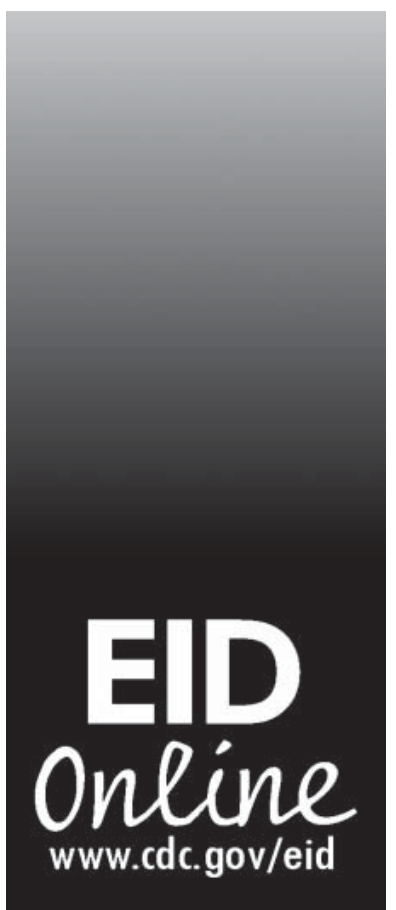

